\title{
A IMPORTÂNCIA DA CULTURA NA INFÂNCIA: UMA PERSPECTIVA TERAPÊUTICO OCUPACIONAL
}

Anne Carolina de Carvalho Morais

Graduanda em Terapia Ocupacional pela Universidade Federal do Pará. E-mail: annemorais97@ gmail.com

Renata Raiol Magalhães

Graduanda em Terapia Ocupacional pela Universidade Federal do Pará. E-mail: gevurah00@yahoo.com.br

\section{RESUMO}

Crianças em situação de vulnerabilidade social podem ter a saúde física, mental e social prejudicada. O contexto cultural influencia diretamente na construção da identidade do indivíduo, bem como suas escolhas. $O$ terapeuta ocupacional pode fomentar o empoderamento e o sentimento de pertencimento e cidadania da criança. Trata-se de um relato de experiência realizado em uma escola de Belémdurante o Estágio Supervisionado I do curso de Terapia Ocupacional da Universidade Federal do Pará. Participou das intervenções, crianças do ensino infantil. Foram realizadas quatro intervenções com duração média de 1 hora. Como instrumentos da pesquisa foram utilizados a anamnese, a ficha de avaliação terapêutica ocupacional e as informações das evoluções dos atendimentos. Foi observado carência em relação ao conhecimento cultural local, e dificuldade na realização de atividades de lazer e participação social. Durante as intervenções, fomentou-se o conhecimento sobre a cultura e o lazer da cidade de Belém, o empoderamento, a formação da identidade pessoal, o sentimento de pertencimento e cidadania, instigando-os a posicionarem como indivíduos ativos na sociedade, estimulou-se a participação e interação social. Utilizou-se contação de história, música, vídeos e passeio. Observou-se que as crianças demonstraram maior interesse pela própria cultura e estabeleceram comunicação e interações sociais respeitosas com os colegas e na família. Crianças em situação de vulnerabilidade social, podem apresentar dificuldades na participação social. A abordagem terapêutica por meio de atividades direcionadas ao contexto cultural podem favorecer o sentimento de pertencimento e cidadania, principalmente das crianças. Portanto o contexto cultural influencia na construção da identidade do indivíduo e na escolha das atividades, estimulando-os a serem agente da própria ação e discurso.

PALAVRAS-CHAVE: Cultura. Infância. Terapia Ocupacional. 


\title{
THE IMPORTANCE OF CULTURE IN CHILDHOOD: AN OCCUPATIONAL THERAPEUTIC PERSPECTIVE
}

\begin{abstract}
Children in a situation of social vulnerability may have impaired physical, mental and social health. The cultural context directly influences the construction of the individual's identity, as well as his choices. The occupational therapist can foster the child's empowerment and sense of belonging and citizenship. This is an experience report carried out at a school in Belém during Supervised Internship I of the Occupational Therapy course at the Federal University of Pará. Children in kindergarten participated in the interventions. Four interventions were performed with an average duration of 1 hour. Anamnesis, the occupational therapeutic assessment form and information on the evolution of care were used as research instruments. There was a shortage in relation to local cultural knowledge, and difficulty in carrying out leisure activities and social participation. During the interventions, knowledge about the culture and leisure of the city of Belém was fostered, empowerment, the formation of personal identity, the feeling of belonging and citizenship, instigating them to position themselves as active individuals in society, was stimulated participation and social interaction. Storytelling, music, videos and walking were used. It was observed that the children showed greater interest in their own culture and established respectful communication and social interactions with colleagues and family. Children in a situation of social vulnerability, may experience difficulties in social participation. The therapeutic approach through activities directed to the cultural context can favor the feeling of belonging and citizenship, especially of children. Therefore, the cultural context influences the construction of the individual's identity and the choice of activities, encouraging them to be agents of their own action and discourse.
\end{abstract}

KEYWORDS: Culture. Childhood. Occupational Therapy

\section{INTRODUÇÃO}

O presente estudo surgiu das reflexões a partir das atividades desenvolvidas durante o Estágio Supervisionado I do curso de Terapia Ocupacional da Universidade Federal do Pará, que tiveram como foco intervenções terapêuticas ocupacionais voltadas às crianças de uma turma do ensino infantil que frequentam uma Instituição de Ação Social.

Crianças em situação de vulnerabilidade social podem ter a saúde física, mental e social prejudicada, visto que se encontram em condição de risco que estão vinculadas diretamente a fatores sociais (ALVES; SANTOS; SANTOS, 2016). Através das relações estabelecidas com o meio e outros seres humanos, que nos apropriamos e internalizamos a cultura local da qual fazemos parte (DILLENBURG; COSTAS; SILVA; WÜRFEL, 2017). Nesse sentido, a criança,
Complexitas - Rev. Fil. Tem., Belém, v. 4, n. 1, p. 18-23, jan./jun. 2019-ISSN: 2525-4154 
que também é um indivíduo inserido na sociedade, sofre com as estruturas sociais oferecidas a ela (ALVES; SANTOS; SANTOS, 2016).

O contexto cultural pode ser compreendido como a construção de significados, partilhados por outros ou não, sustentados em práticas da vida individual e social, influenciando diretamente na construção da identidade do indivíduo, e nas suas escolhas, além de ser retrato de um patrimônio imaterial valioso, que reflete a história do indivíduo e da sociedade (BRASIL, 2019; AOTA, 2015; BARBOSA, 2007).

A realização de atividades culturais durante a infância é fundamental, visto que o desenvolvimento das crianças não é algo individual, mas um processo cultural e, portanto, coletivo que acontece continuamente através das relações de brincadeira e de faz-de-conta desenvolvidas pelas crianças (BARBOSA, 2007). Além disso, as atividades culturais da vida adulta sofrem influência das práticas culturais exercidas durante a infância. Indivíduos que na infância, praticaram atividades como: tocar instrumento, leitura, visita ao museu, teatro, cinema, tem mais probabilidade de realiza-las ao longo do ano (BARBOSA, 2007).

Nesse sentido, se faz necessário permitir que as crianças se tornem agentes de sua própria ação e discurso, considerando-as aptas a organizar suas vidas e estimulando-as a contribuir ativamente para a mudança cultural da sociedade (BARBOSA, 2007).

Profissionais que estão relacionados de alguma forma ao processo de desenvolvimento infantil, assim como o terapeuta ocupacional, podem contribuir na produção de novas culturas, fazer dialogar, interagir, comunicar as culturas, criando um espaço intercultural (BARBOSA, 2007).

O terapeuta ocupacional pode fomentar o empoderamento e o sentimento de pertencimento e cidadania da criança, valorizar a dimensão do lúdico, da imaginação, da experimentação do mundo pelo brinquedo e a brincadeira, de modo a garantir o desenvolvimento pleno das crianças (BRASIL, 2019).

Este estudo teve como objetivo possibilitar reflexões referentes a atuação terapêutica ocupacional no contexto cultural do indivíduo, principalmente durante a infância, visto que nessa fase o sujeito está em formação da identidade pessoal, social e cultural, bem como a importância desse processo no sentimento de pertencimento e cidadania do indivíduo, e na modificação das estruturas sociais.

\section{A TERAPIA OCUPACIONAL E A CULTURA NA INFÂNCIA}


O terapeuta ocupacional, durante suas pratica tem a possibilidade de ampliar as experiências culturais das crianças, socializando os seus saberes e conhecimentos, estimulando o diálogo intercultural, favorecer a expressão das crianças em todas as formas, valorizando as diferentes linguagens e expressões (MEC, 2016).

A criança como um indivíduo inserido na sociedade, tem a possibilidade de modificar as estruturas sociais em que se encontram, ressignificando-as por meio de suas produções singulares, criativas e imprevisíveis (MEC, 2016). Nesse sentido, as crianças participam ativamente da cultura, estabelecendo com outros sujeitos interações efetivas que são inerentes às relações humanas.

Durante o estágio na Instituição de Ação Social foram realizadas quatro intervenções com duração média de 1 hora. Como instrumentos da pesquisa foram utilizados a anamnese, a ficha de avaliação terapêutica ocupacional e as informações das evoluções dos atendimentos. Foi observado carência em relação ao conhecimento cultural local, e dificuldade na realização de atividades de lazer e participação social.

De acordo com MEC (2016) as crianças aprendem e conhecem a cultura humana ciência, arte e vida - no fazer, agir e interagir, principalmente quando ocorre de forma lúdica. Nesse sentido, durante as intervenções, fomentou-se o conhecimento sobre a cultura e o lazer da cidade de Belém, o sentimento de pertencimento e cidadania, instigando-os a posicionarem como indivíduos ativos na sociedade, estimulou-se a participação e interação social, utilizando brincadeiras, contação de história, música, vídeos e passeio que apresentam o mundo ficcional e não ficcional (MEC, 2016).

No primeiro encontro foi realizado as avaliações e observado e/ou relatado pelas professoras as demandas das crianças. Também foi proposto atividade de contação de histórias com fantoches, para iniciar o primeiro contato entre as discentes e as crianças, e favorecer o vínculo.

Na segunda intervenção foi realizado uma apresentação com fotos, vídeos, e brincadeiras relacionadas as lendas da Amazonia. Algumas lendas como Iara, Boto, Curupira eram conhecidas pelas crianças, outras como a Vitória-régia, Macunaíma, não faziam parte do repertório.

A terceira intervenção teve como foco os pontos turísticos e culturais de Belém, como o Teatro da Paz, Praça Batista Campos, Museu Emílio Goeldi, Bosque Rodrigues Alves, Centro Cultural e Turístico Tancredo Neves (CENTUR), entre outros. Além de apresentar os locais, foi 
enfatizado a importância de a criança frequentá-los pois dessa forma estariam realizando ocupações importantes para o desenvolvimento, como o brincar e a participação social.

Em relação ao CENTUR, foi mencionado a presença da brinquedoteca e da gibiteca que podem ser usufruídos de forma gratuita, estimulando assim a realização do brincar, pois grande parte das crianças da Instituição de Ação Social se encontram em situação socioeconômica vulnerável, e apresentaram prejuízo na realização do brincar, por alguns fatores como falta de recursos, por despender tempo nas tarefas da casa, no cuidado com parentes mais novos.

Na última intervenção foi realizado uma visita guiada ao Museu Emilio Goeldi, no qual a monitora comentava informações e curiosidades sobre o local e os animais que ali residem. As crianças demonstraram interesse, satisfação e contentamento com a atividade, mostrando-se comunicativos durante a visita, e relacionando com o diálogo realizado na intervenção anterior.

Observou-se que as crianças demonstraram maior interesse pela própria cultura e estabeleceram comunicação e interações sociais respeitosas com os colegas e na família. O estímulo ao diálogo intercultural favorece a participação social da criança, e desse modo, promove mudanças nas estruturas sociais estabelecidas, as crianças demonstraram uma visão mais crítica no que concerne ao meio em que vive e as relações que são estabelecidas

\section{CONSIDERAÇÕES FINAIS}

Crianças em situação de vulnerabilidade social, podem apresentar dificuldades na participação social. A abordagem terapêutica por meio de atividades direcionadas ao contexto cultural podem favorecer o sentimento de pertencimento e cidadania da criança, contribuindo ativamente para a mudança cultural da sociedade, valorizar a dimensão do lúdico, da imaginação, auxiliar na a formação da identidade pessoal, e estimular a participação e interação social. Portanto o contexto cultural influencia na construção da identidade do indivíduo e na escolha das atividades, estimulando-os a serem agente da própria ação e discurso.

\section{REFERÊNCIAS BIBLIOGRÁFICAS}

ALVES, Layane Pereira; SANTOS, 68, 2016.

Vinicius Silva; SANTOS, Jacques

Fernandes. $\quad$ INFÂNCIA,

AOTA AMERICAN OCCUPATIONAL

VULNERABILIDADE E SITUAÇÃO DE THERAPY ASSOCIATION et al.

RISCO EM PAULO AFONSO- Estrutura da prática da Terapia

BAHIA. Revista Científica da FASETE, p. Ocupacional: domínio \& processo- 
traduzida. Revista de Terapia Ocupacional da Universidade de São Paulo, v. 26, p. 149, 2015.

\section{BARBOSA, Maria Carmen Silveira.}

Culturas escolares, culturas de infância e culturas familiares: as socializações e a escolarização no entretecer destas culturas. Educação \& Sociedade, v. 28, n. 100, p. 1059-1083, 2007.

BRASIL. Ministério da Cidadania. Jogos $e$ brincadeiras das culturas populares na Primeira Infância / Ministério da Cidadania.

- Brasília: Ministério da Cidadania, 2019.
T., SILVA, L. C. D., WÜRFEL, R. F. Vulnerabilidade social e desenvolvimento infantil: um olhar a partir da Teoria Histórico Cultural e da Neurociência. Revista Educação e Cultura Contemporânea, v. 14, n. 36, p. 279-295, 2017.

MEC. Crianças como leitoras e autoras / Ministério da Educação, Secretaria de Educação Básica.- 1.ed.- Brasília: MEC /SEB, 2016.128 p.: il.; 20,5 x 27,5 cm.(Coleção Leitura e escrita na educação infantil ; v.6).

DILlENBURG, A. I., COSTAS, F. A.

MORAIS. A.C.C., MAGALHÃES, R. R. A Importância da Cultura na Infância: Uma Perspectiva Terapêutico Ocupacional. Complexitas - Rev. Fil. Tem. Belém, v. 4, n. 1, p. 18-23, jan./jun. 2019. Disponível em: http://www.periodicos.ufpa.br/index.php/complexitas/article/view/8040>. Acesso em: 30 de janeiro de 2020 . 